Pacific Journal of Mathematics

DENSITY OF THE POLYNOMIALS IN BERGMAN SPACES 


\title{
DENSITY OF THE POLYNOMIALS IN BERGMAN SPACES
}

\author{
PAul S. Bourdon
}

Let $G$ be a bounded simply connected domain in the complex plane. Using a result of Hedberg, we show that the polynomials are dense in Bergman space $L_{a}^{2}(G)$ if $G$ is the image of the unit disk $U$ under a weak-star generator of $H^{\infty}$. We also show that density of the polynomials in $L_{a}^{2}(G)$ implies density of the polynomials in $H^{2}(G)$. As a consequence, we obtain new examples of cyclic analytic Toeplitz operators on $H^{2}(U)$ and composition operators with dense range on $H^{2}(U)$. As an additional consequence, we show that if the polynomials are dense in $L_{a}^{2}(G)$ and $\varphi$ maps $U$ univalently onto $G$, then $\varphi$ is univalent almost everywhere on the unit circle $C$.

1. Introduction. Let $\Omega$ be an open, nonempty subset of the complex plane, and let $d A$ be two-dimensional Lebesgue measure. The Bergman space of $\Omega, L_{a}^{2}(\Omega)$, is the Hilbert space of those functions $f$ which are analytic on $\Omega$ and which satisfy

$$
\|f\|^{2}=\int_{\Omega}|f|^{2} d A<\infty .
$$

Let $H^{\infty}$ denote the algebra of functions which are bounded and analytic on the open unit disk $U$.

For any domain $G$ in the plane, define the Carathéodory hull of $G$, $G^{*}$, to be the complement of the closure of the unbounded component of the complement of the closure of $G$. If $G$ is a component of its Carathéodory hull $G^{*}$, then $G$ is said to be a Carathéodory domain. Carathéodory domains are simply connected (cf. for example [14, Lemma 2.13]). An old theorem (1934) of Farrell [6] and Markusevic [8] states that if $G$ is a bounded Carathéodory domain, then the polynomials are dense in $L_{a}^{2}(G)$. In 1953, S. N. Mergeljan remarked in his survey article on polynomial approximation that the Carathéodory domains apparently form the largest class of domains $G$ with a purely topological definition such that the polynomials are dense in $L_{a}^{2}(G)$ (cf. [9, p. 121]). We show that there is a larger class of such domains.

THEOREM 3.1. If $G=\varphi(U)$ where $\varphi$ is a weak-star generator of $H^{\infty}$, then the polynomials are dense in $L_{a}^{2}(G)$. 
The function $\varphi \in H^{\infty}$ is a weak-star generator of $H^{\infty}$ provided the polynomials in $\varphi$ are weak-star dense in $H^{\infty}$. Here, it will be convenient to view $H^{\infty}$ as the dual of a quotient space of $L^{1}(U)$. (The predual of $H^{\infty}$ is, in fact, unique [2, Theorem 1].) In [16] Sarason characterizes the images of weak-star generators of $H^{\infty}$ using the concept of relative hulls. Proposition 4 of [16] shows that relative hulls have a topological description based on the notion of a crosscut. Theorem 3.1 extends the result of Farrell and Markusevic since if $\varphi$ maps $U$ univalently onto a bounded Carathéodory domain, then $\varphi$ is a weak-star generator of $H^{\infty}$ (of order 1) [16]. Moreover, there are many weak-star generators of $H^{\infty}$ which map $U$ onto non-Carathéodory domains $[\mathbf{1 6}, \mathbf{1 7}]$.

Weak-star generators of $H^{\infty}$ are univalent on $U$ and univalent almost everywhere on the unit circle $C$ [15, Propositions 2 and 3]. We show that for the polynomials to be dense in $L_{a}^{2}(G)$, it is necessary that the univalent map of $U$ onto $G$ be univalent a.e. on $C$. This gives another way to see that if, for example, $G$ contains slits (which are not too close together) then the polynomials are not dense in $L_{a}^{2}(G)$. Here, and for the remainder of this paper, we use the letter $G$ to denote a bounded simply connected domain in the plane.

2. Preliminaries. Let $A^{2}$ denote the Bergman space of the unit disk; that is, let $A^{2}=L_{a}^{2}(U)$. For $f \in H^{\infty}$, define $B_{f}: A^{2} \rightarrow A^{2}$ by

$$
\left(B_{f} g\right)(z)=f(z) g(z) \text {. }
$$

Similarly, define $T_{f}: H^{2}(U) \rightarrow H^{2}(U)$ by $\left(T_{f} h\right)(z)=f(z) h(z)$; here, $H^{2}(U)$ denotes the Hardy space of $U$. For any operator $A$, let Lat $A$ represent the lattice of invariant subspaces of $A$ (subspace $\equiv$ closed subspace). Lat $T_{\varphi}=$ Lat $T_{z}$ if and only if $\varphi$ is a weak-star generator of $H^{\infty}$ [15, Proposition 1].

Proposition 2.1. If $\varphi$ is a weak-star generator of $H^{\infty}$, then Lat $B_{\varphi}=$ Lat $B_{z}$.

Proof. That Lat $B_{z} \subset$ Lat $B_{\varphi}$ for any $\varphi \in H^{\infty}$ is well known (cf. for example [19, Theorem 12]). If $\varphi$ is a weak-star generator of $H^{\infty}$ then there is a net $\left\{p_{\alpha}\right\}$ of polynomials such that $p_{\alpha}(\varphi) \rightarrow z$ weak-star in $H^{\infty}$. This means that for any $f \in L^{1}(U)$

$$
\int_{U}\left(p_{\alpha}(\varphi)-z\right) f d A \rightarrow 0 .
$$

It follows that $B_{p_{\alpha}(\varphi)} \rightarrow B_{z}$ in the weak operator topology. Hence, Lat $B_{\varphi}$ $\subset$ Lat $B_{z}$. 
John Conway and Robert Olin have pointed out to the author that the converse of Proposition 2.1 is true. If Lat $B_{\varphi}=$ Lat $B_{z}$, then by the reflexivity of subnormal operators, there is a net $\left\{p_{\alpha}\right\}$ of polynomials such that $B_{p_{\alpha}(\varphi)} \rightarrow B_{z}$ in the weak operator topology. That $p_{\alpha}(\varphi) \rightarrow z$ weak-star follows from Theorems 1 and 2 of [12].

The proof of the following theorem appears in [19, pp. 112-114].

THEOREM 2.2 (Hedberg). If $R$ is a simply connected domain of finite area, then $H^{\infty}(R)$ is dense in $L_{a}^{2}(R)$.

Via a change of variables, Hedberg's result is equivalent to the following (cf. [19, Proposition 41]).

COROLlary 2.3. If $f$ maps $U$ univalently onto a domain of finite area, then the derivative of $f$ is cyclic for $B_{z}$.

The vector $g \in A^{2}$ is cyclic for $B_{f}: A^{2} \rightarrow A^{2}$ provided $g$ is not contained in any proper invariant subspace of $B_{f}$. Alternatively, $g$ is cyclic for $B_{f}$ if $\{p(f) g: p$ is a polynomial $\}$ is dense in $A^{2}$.

3. Results. One may combine Corollary 2.3 and Proposition 2.1 to obtain a simple proof of Theorem 3.1.

THEOREM 3.1. If $G=\varphi(U)$ where $\varphi$ is a weak-star generator of $H^{\infty}$, then the polynomials are dense in $L_{a}^{2}(G)$.

Proof. Since weak-star generators are univalent, $\varphi^{\prime}$ is cyclic for $B_{z}$; and since Lat $B_{\varphi}=$ Lat $B_{z}, \varphi^{\prime}$ is cyclic for $B_{\varphi}$.

Now, let $g \in L_{a}^{2}(G)$ be arbitrary. Since $g(\varphi) \varphi^{\prime} \in L_{a}^{2}(U)=A^{2}$ and since $\varphi^{\prime}$ is cyclic for $B_{\varphi}$, there is a sequence $\left\{p_{n}\right\}$ of polynomials such that

$$
\int_{U}\left|p_{n}(\varphi) \varphi^{\prime}-g(\varphi) \varphi^{\prime}\right|^{2} d A \rightarrow 0 \text {. }
$$

Changing variables, we have $\int_{G}\left|p_{n}-g\right|^{2} d A \rightarrow 0$. Hence, the polynomials are dense in $L_{a}^{2}(G)$.

Robert Olin has related to the author another argument which yields Theorem 3.1. The author wishes to thank Prof. Olin for his permission to give that argument here. Let $S$ be a bounded open set in the plane and let $\tilde{K}$ be its Sarason hull. ( $\tilde{K}$ is the Sarason hull of $\mu$ where $\mu$ is two-dimensional Lebesgue measure on $S$. See [18].) The polynomials are weak-star dense in $H^{\infty}(S)$ if and only if every function in $H^{\infty}(S)$ extends to a 
function in $H^{\infty}$ (int $\tilde{K}$ ) [18, Corollary 3]. The following proposition holds since if $G$ is the image of a weak-star generator of $H^{\infty}$, then $G$ is the interior of its Sarason hull $\tilde{K}$ (cf. [11, Lemma 1]).

Proposition 3.2. If $G=\varphi(U)$ where $\varphi$ is a weak-star generator of $H^{\infty}$, then the polynomials are weak-star dense in $H^{\infty}(G)$.

Here, $H^{\infty}(G)$ is viewed as the dual of a quotient space of $L^{1}(G)$. If $G$ is the image of a weak-star generator, then it follows easily from Proposition 3.2 that $H^{\infty}(G)$ is contained in weak closure of the polynomials in $L_{a}^{2}(G)$. Since for a convex subset of a Banach space weak closure is equivalent to norm closure, $H^{\infty}(G)$ is contained in the norm closure of the polynomials in $L_{a}^{2}(G)$. Theorem 3.1 now follows from Hedberg's result (Theorem 2.2).

There is no problem extending Hedberg's result to a bounded open set each of whose components is simply connected. Hence, Olin's argument provides a generalization of Theorem 3.1: If $S$ is a bounded open subset of the plane with Sarason hull $\tilde{K}$, then the polynomials are dense in $L_{a}^{2}(S)$ if each $f \in L_{a}^{2}(S)$ extends to a function $f \in L_{a}^{2}($ int $\tilde{K})$. One way to see that the components of int $\tilde{K}$ are simply connected is to combine Lemma 7.1 of [18] with Theorem 5.1 of [7]. This generalization of Theorem 3.1 provides an extension of a result of Sinanjan [20] who showed that the polynomials are dense in $L_{a}^{2}(S)$ if $S$ is a bounded Carathéodory set. ( $S$ is a Carathéodory set provided it is the union of some of the components of $S^{*}$.) Rubel and Shields have actually shown that for a bounded Carathéodory set $S$ the polynomials are weak-star sequentially dense in $H^{\infty}(S)$; that is, each $f \in H^{\infty}(S)$ is the pointwise limit of a uniformly bounded sequence of polynomials (cf. [14, Theorem 3.2]). Note that it's a simple matter to combine the result of Rubel and Shields with Hedberg's result to obtain Sinanjan's result.

We turn now to a proposition which yields a necessary condition for the polynomials to be dense in $L_{a}^{2}(G)$ and which provides new examples of cyclic analytic Toeplitz operators on $H^{2}(U)$ and composition operators with dense range on $H^{2}(U)$. For any positive Borel measurable function $w$ on $G$, let $L_{a}^{2}(G, w d A)$ represent the weighted Bergman space consisting of those analytic functions $f$ on $G$ which satisfy

$$
\|f\|_{w}^{2}=\int_{G}|f|^{2} w d A<\infty .
$$

Proposition 3.3. Let $\varphi$ map $U$ univalently onto $G$. The polynomials are dense in $L_{a}^{2}\left(G,\left(1-\left|\varphi^{-1}(z)\right|^{2}\right) d A\right)$ if and only if the polynomials in $\varphi$ are dense in $H^{2}(U)$. 
Proof. Recall that for $f=\sum_{n=0}^{\infty} \hat{f}(n) z^{n} \in H^{2}(U)$,

$$
\|f\|_{H^{2}(U)}^{2}=\sum_{n=0}^{\infty}|\hat{f}(n)|^{2}=\frac{1}{2 \pi} \int_{0}^{2 \pi}\left|f\left(e^{i \theta}\right)\right|^{2} d \theta
$$

where for almost every $\theta, f\left(e^{\imath \theta}\right)=\lim _{r \rightarrow 1} f\left(r e^{\imath \theta}\right)$. By considering the Taylor expansion of $f \in H^{2}(U)$, one may easily verify that \|\|$_{H^{2}(U)}$ is equivalent to || || where

$$
\|f\|^{2}=|f(0)|^{2}+\int_{U}\left|f^{\prime}\right|^{2}\left(1-|z|^{2}\right) d A
$$

Now if the polynomials are dense in $L_{a}^{2}\left(G,\left(1-\left|\varphi^{-1}(z)\right|^{2}\right) d A\right)$, then $\left\{p(\varphi) \varphi^{\prime}: p\right.$ is a polynomial $\}$ is dense in $L_{a}^{2}\left(U,\left(1-|z|^{2}\right) d A\right)$. Integrating, we see that $\{p(\varphi): p$ is a polynomial $\}$ is dense in $H^{2}(U)$. The converse follows by differentiating.

This simple proposition has several interesting consequences. The following consequence seems to have been overlooked in the literature. It shows that, for example, a result of Caughran (cf. [4, Theorem 1]) is actually an easy consequence of the result of Farrell and Markusevic. Recall that a function $f$ belongs to the Hardy space $H^{2}(G)$ provided $|f|^{2}$ has a harmonic majorant on $G$. One defines the norm of $f \in H^{2}(G)$ by $\|f\|_{H^{2}(G)}=\left[u\left(z_{0}\right)\right]^{1 / 2}$, where $z_{0}$ is a fixed point in $G$ and $u$ is the least harmonic majorant of $|f|^{2}$. If $\varphi$ maps $U$ univalently onto $G$ with $\varphi(0)=z_{0}$ then the correspondence $f \leftrightarrow f(\varphi)$ is an isometric isomorphism between $H^{2}(G)$ and $H^{2}(U)$ (normed as in the proof of Proposition 3.3) (cf. for example [5, Chapter 10]).

Corollary 3.4. Let $\varphi$ map $U$ univalently onto $G$. Density of the polynomials in $L_{a}^{2}(G)$ (or in $L_{a}^{2}\left(G,\left(1-\left|\varphi^{-1}(z)\right|^{2}\right) d A\right)$ ) implies density of the polynomials in $H^{2}(G)$.

Proof. It's easy to see via a change of variables that density of the polynomials in $L_{a}^{2}(G)$ implies density of the polynomials in $L_{a}^{2}\left(G,\left(1-\left|\varphi^{-1}(z)\right|^{2}\right) d A\right)$. By Proposition $3.3,\{p(\varphi): p$ is a polynomial $\}$ is dense in $H^{2}(U)$, but this is equivalent to density of the polynomials in the Hardy space $H^{2}(G)$.

COROllary 3.5. If $\varphi$ maps $U$ univalently onto $G$ and if the polynomials are dense in $L_{a}^{2}(G)$ (or in $L_{a}^{2}\left(G,\left(1-\left|\varphi^{-1}(z)\right|^{2}\right) d A\right)$ ), then $\varphi$ is univalent almost everywhere on the unit circle $C$. 
Proof. By Proposition 3.3, $\{p(\varphi): p$ is a polynomial $\}$ is dense in $H^{2}(U)$; in particular, there is a sequence $\left\{p_{n}\right\}$ of polynomials such that $\left\|p_{n}(\varphi)-z\right\|_{H^{2}(U)} \rightarrow 0$. Choose a subsequence $\left\{p_{n_{j}}\right\}$ of $\left\{p_{n}\right\}$ such that $p_{n_{j}}(\varphi(z)) \rightarrow z$ a.e. on $C$. Off the set of measure zero on which $p_{n_{j}}(\varphi(z))$ may not go to $z, \varphi$ must be univalent.

The following two corollaries are immediate consequences of Proposition 3.3.

Corollary 3.6. If $\varphi$ maps $U$ univalently to $G$ and if the polynomials are dense in $L_{a}^{2}\left(G,\left(1-\left|\varphi^{-1}(z)\right|^{2}\right) d A\right)$, then the analytic Toeplitz operator $T_{\varphi}: H^{2}(U) \rightarrow H^{2}(U)$ is cyclic with cyclic vector 1 .

If $\varphi$ is a weak-star generator of $H^{\infty}$, then it's easy to see (for example, by using $\operatorname{Lat} T_{z}=\operatorname{Lat} T_{\varphi}$ ) that $T_{\varphi}$ is cyclic with cyclic vector 1 . John Akeroyd [1] has produced examples of cyclic analytic Toeplitz operators whose symbols are not weak-star generators. In fact, he has shown that if $\varphi$ maps $U$ univalently onto a crescent bounded by two internally tangent circles, then $T_{\varphi}$ is cyclic with cyclic vector 1 . That a crescent is not the image of a weak-star generator follows from [16, Corollary 2]. Corollary 3.6 above provides further examples of cyclic analytic Toeplitz operators. There are bounded simply connected domains $G$ such that the polynomials are dense in $L_{a}^{2}(G)$ (hence in $L_{a}^{2}\left(G,\left(1-\left|\varphi^{-1}(z)\right|^{2}\right) d A\right)$ ), but $G$ is not the image of a weak-star generator. For example, Mergeljan and Tamadjan [10] (cf. also [3]) have shown that if sufficiently many slits are put in the unit disk, one can obtain a domain $G$ such that the polynomials are dense in $L_{a}^{2}(G)$. Once again, Corollary 2 of [16] shows that the disk with these slits is not the image of a weak-star generator of $H^{\infty}$.

COROllary 3.7. Let $\varphi$ map $U$ univalently onto $G \subset U$ and define $C_{\varphi}$ : $H^{2}(U) \rightarrow H^{2}(U)$ by $\left(C_{\varphi} f\right)(z)=f(\varphi(z))$. If the polynomials are dense in $L_{a}^{2}\left(G,\left(1-\left|\varphi^{-1}(z)\right|^{2}\right) d A\right)$ then $C_{\varphi}$ has dense range.

Corollary 3.7 extends a result of Roan [13] by providing additional examples of composition operators with dense range.

ReMarK. It's easy to see that if $C_{\varphi}$ has dense range or if $T_{\varphi}$ is cyclic, then $\varphi$ is univalent on $U$ and univalent a.e. on $C$.

\section{REFERENCES}

[1] J. Akeroyd, Approximation in the mean by polynomials with respect to harmonic measure, preprint.

[2] T. Ando, On the predual of $H^{\infty}$, Comment. Math. Spec. Issue, 1 (1978), 33-40. 
[3] J. E. Brennan, Approximation in the mean by polynomials on non-Carathéodory domains, Ark. Mat., 15 (1977), 117-168.

[4] J. Caughran, Polynomial approximation and spectral properties of composition operators on $H^{2}$, Indiana Univ. Math. J., 21 (1971), 81-84.

[5] P. L. Duren, Theory of $H^{p}$ Spaces, Academic Press, New York, 1970.

[6] O. J. Farrell, On approximation to an analytic function by polynomials, Bull. Amer. Math. Soc., 40 (1934), 908-914.

[7] T. W. Gamlin and J. Garnett, Pointwise bounded approximation in Dirichlet algebras, J. Functional Anal., 8 (1971), 360-404.

[8] A. I. Markusevic, Conformal mapping of regions with variable boundary and application to the approximation of analytic functions by polynomials, Dissertation, Moscow, 1934.

[9] S. N. Mergeljan, On the completeness of systems of analytic functions, Amer. Math. Soc. Transl., 19 (1962), 109-166; Uspekhi Mat. Nauk, 8 (1953), 3-63.

[10] S. N. Mergeljan and A. P. Talmadjan, On completeness in a class of non-Jordan regions, Amer. Math. Soc. Transl., 35 (1964), 79-94; Izv. Akad. Nauk Armjan. SSR, 7 (1954), 1-17.

[11] R. Olin and J. Thomson, Lifting the commutant of a subnormal operator, Canad. J. Math., 31 (1979), 148-156.

[12] _ Algebras of subnormal operators, J. Functional Anal., 37 (1980), 271-301.

[13] R. C. Roan, Composition operators on $H^{p}$ with dense range, Indiana Univ. Math. J., 27 (1978), 159-162.

[14] L. Rubel and A. L. Shields, Bounded approximation by polynomials, Acta Math., 112 (1964), 145-162.

[15] D. Sarason, Invariant subspaces and unstarred operator algebras, Pacific J. Math., 17 (1966), 511-517.

[16] _ Weak-star generators of $H^{\propto}$, Pacific J. Math., 17 (1966), 519-528.

[17] __, On the order of a simply connected domain, Michigan Math. J., 15 (1968), 129-133.

[18] __ Weak-star density of polynomials, J. Reine Angew. Math., 252 (1972), 1-15.

[19] A. L. Shields, Weighted shift operators and analytic function theory, Math. Surveys, 13: Topics in operator theory, ed. C. Pearcy, Amer. Math. Soc., Providence, R. I., 1974, 49-128.

[20] S. O. Sinanjan, Approximation by polynomials and analytic functions in the areal mean, Amer. Math. Soc. Transl., 74 (1968), 91-124; Mat. Sb., 69 (1966), 546-578.

Received February 24, 1986 and in revised form February 23, 1987.

Michigan State University

EAST LANSING, MI 48824 



\section{EDITORS}

\author{
V. S. VARADARAJAN \\ (Managing Editor) \\ University of California \\ Los Angeles, CA 90024 \\ HeRbert Clemens \\ University of Utah \\ Salt Lake City, UT 84112 \\ R. FINN \\ Stanford University \\ Stanford, CA 94305
}

\author{
HERMANN FLASCHKA \\ University of Arizona \\ Tucson, AZ 85721 \\ RAMESh A. GANGOLLI \\ University of Washington \\ Seattle, WA 98195 \\ VAUGHAN F. R. JONES \\ University of California \\ Berkeley, CA 94720
}

\author{
ROBION KIRBY \\ University of California \\ Berkeley, CA 94720 \\ C. C. MOORE \\ University of California \\ Berkeley, CA 94720 \\ HAROLD STARK \\ University of California, San Diego \\ La Jolla, CA 92093
}

\section{ASSOCIATE EDITORS}
R. ARENS
E. F. BECKENBACH
B. H. NEUMANN
F. WOLF
K. YOSHIDA
(1906-1982)

\section{SUPPORTING INSTITUTIONS}

UNIVERSITY OF ARIZONA
UNIVERSITY OF BRITISH COLUMBIA
CALIFORNIA INSTITUTE OF TECHNOLOGY
UNIVERSITY OF CALIFORNIA
MONTANA STATE UNIVERSITY
UNIVERSITY OF NEVADA, RENO
NEW MEXICO STATE UNIVERSITY
OREGON STATE UNIVERSITY

\author{
UNIVERSITY OF OREGON \\ UNIVERSITY OF SOUTHERN CALIFORNIA \\ STANFORD UNIVERSITY \\ UNIVERSITY OF HAWAII \\ UNIVERSITY OF TOKYO \\ UNIVERSITY OF UTAH \\ WASHINGTON STATE UNIVERSITY \\ UNIVERSITY OF WASHINGTON
}

The Supporting Institutions listed above contribute to the cost of publication of this Journal, but they are not owners or publishers and have no responsibility for its content or policies.

Mathematical papers intended for publication in the Pacific Journal of Mathematics should be in typed form or offset-reproduced (not dittoed), double spaced with large margins. Please do not use built up fractions in the text of the manuscript. However, you may use them in the displayed equations. Underline Greek letters in red, German in green, and script in blue. The first paragraph must be capable of being used separately as a synopsis of the entire paper. In particular it should contain no bibliographic references. Please propose a heading for the odd numbered pages of less than 35 characters. Manuscripts, in triplicate, may be sent to any one of the editors. Please classify according to the scheme of Math. Reviews, Index to Vol. 39. Supply name and address of author to whom proofs should be sent. All other communications should be addressed to the managing editor, or Elaine Barth, University of California, Los Angeles, California 90024.

There are page-charges associated with articles appearing in the Pacific Journal of Mathematics. These charges are expected to be paid by the author's University, Government Agency or Company. If the author or authors do not have access to such Institutional support these charges are waived. Single authors will receive 50 free reprints; joint authors will receive a total of 100 free reprints. Additional copies may be obtained at cost in multiples of 50 .

The Pacific Journal of Mathematics is issued monthly as of January 1966. Regular subscription rate: $\$ 190.00$ a year (5 Vols., 10 issues). Special rate: $\$ 95.00$ a year to individual members of supporting institutions.

Subscriptions, orders for numbers issued in the last three calendar years, and changes of address should be sent to Pacific Journal of Mathematics, P.O. Box 969, Carmel Valley, CA 93924, U.S.A. Old back numbers obtainable from Kraus Periodicals Co., Route 100, Millwood, NY 10546.

The Pacific Journal of Mathematics at P.O. Box 969, Carmel Valley, CA 93924 (ISSN 0030-8730) publishes 5 volumes per year. Application to mail at Second-class postage rates is pending at Carmel Valley, California, and additional mailing offices. Postmaster: send address changes to Pacific Journal of Mathematics, P.O. Box 969, Carmel Valley, CA 93924.

PUBLISHED BY PACIFIC JOURNAL OF MATHEMATICS, A NON-PROFIT CORPORATION Copyright (c) 1987 by Pacific Journal of Mathematics 


\section{Pacific Journal of Mathematics}

\section{Vol. 130, No. $2 \quad$ October, 1987}

Bernhard Banaschewski, J. L. Frith and C. R. A. Gilmour, On the congruence lattice of a frame ............................ 209

Paul S. Bourdon, Density of the polynomials in Bergman spaces ........2215

Lawrence Jay Corwin, Approximation of prime elements in division

algebras over local fields and unitary representations of the multiplicative group

Stephen R. Doty and John Brendan Sullivan, On the geometry of extensions of irreducible modules for simple algebraic groups

Karl Heinz Dovermann and Reinhard Schultz, Surgery of involutions

with middle-dimensional fixed point set ....................2 275

Ian Graham, Intrinsic measures and holomorphic retracts ............ 299

John Robert Greene, Lagrange inversion over finite fields . . . . . . . . . . . 313

Kristina Dale Hansen, Restriction to $\mathrm{GL}_{2}(\mathrm{O})$ of supercuspidal

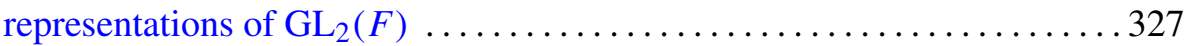

Kei Ji Izuchi, Unitary equivalence of invariant subspaces in the polydisk ....351

A. Papadopoulos and R. C. Penner, A characterization of pseudo-Anosov

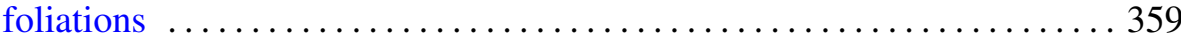

Erik A. van Doorn, The indeterminate rate problem for birth-death

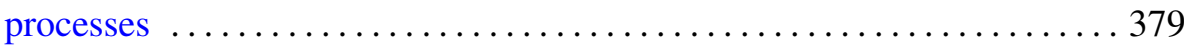

Ralph Jay De Laubenfels, Correction to: "Well-behaved derivations on

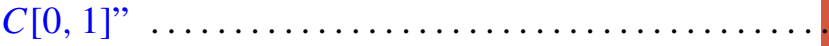

Robert P. Kaufman, Correction to: "Plane curves and removable sets" . . . . 396

Richard Scott Pierce and Charles Irvin Vinsonhaler, Correction to:

"Realizing central division algebras" 